\title{
Multivortex and giant vortex states near the expulsion and penetration fields in thin mesoscopic superconducting squares
}

\author{
B. J. Baelus,,${ }^{1, *}$ A. Kanda, ${ }^{2, \dagger}$ N. Shimizu, ${ }^{2}$ K. Tadano,${ }^{2}$ Y. Ootuka, ${ }^{2}$ K. Kadowaki, ${ }^{3}$ and F. M. Peeters ${ }^{1, \ddagger}$ \\ ${ }^{1}$ Departement Fysica, Universiteit Antwerpen, Groenenborgerlaan 177, B-2020 Antwerpen, Belgium \\ ${ }^{2}$ Institute of Physics and Tsukuba Research Center for Interdisciplinary Materials Science (TIMS), University of Tsukuba, Tsukuba \\ 305-8571, Japan \\ ${ }^{3}$ Institute of Materials Science, University of Tsukuba, Tsukuba 305-8573, Japan
}

(Received 26 September 2005; revised manuscript received 19 December 2005; published 31 January 2006)

\begin{abstract}
The temperature dependence of the vortex penetration and expulsion magnetic fields are investigated, both theoretically and experimentally, for mesoscopic superconducting squares. The small-tunnel-junction method is used to determine the transition fields between the different vortex states. We found that the penetration fields decrease with increasing temperature, while the expulsion fields for a particular vorticity may increase, decrease or be independent of temperature. Using the Ginzburg-Landau theory we link the different temperature dependencies to the configuration of the vortex state, i.e. giant vortex state versus multivortex state. The vortex state consisting of four vortices has a larger stability region which is most pronounced in a certain hightemperature range.
\end{abstract}

DOI: 10.1103/PhysRevB.73.024514

PACS number(s): 74.78.- $-\mathrm{w}, 74.78 . \mathrm{Na}, 74.25 . \mathrm{Op}$

\section{INTRODUCTION}

When the sizes of superconducting samples become of the order of the coherence length $\xi$ or the penetration depth $\lambda$, the sample boundary drastically influences the vortex structure, which may strongly deviate from the triangular Abrikosov vortex lattice. For such mesoscopic superconductors, it is known that the vortex arrangement is determined by a competition between the vortex-vortex interaction and the boundary that tries to impose its symmetry on the vortex configuration (see, e.g., Refs. 1 and 2).

During the last decade, the study of mesoscopic superconductors has attracted a lot of attention. Circular mesoscopic disks have been the most popular in this respect, both theoretically ${ }^{1-6}$ and experimentally. ${ }^{7-13}$ Two types of vortex states were found in such mesoscopic superconducting disks: (i) giant vortex states (GVSs), where the order parameter has a single zero and (ii) multivortex states (MVSs) consisting of several singly quantized vortices (mostly situated on shells). Experimentally, one measured the resistivity ${ }^{7,8}$ and the magnetization ${ }^{9,10}$ of the superconducting disk for the different vortex states. Since these quantities do not provide direct information on the vortex configuration, there was no direct proof for the existence of the different types of vortex states in mesoscopic superconductors.

Recently, we achieved an important breakthrough in the experimental study of vortices in mesoscopic superconducting disks by using the multiple-small-tunnel-junction (MSTJ) method, ${ }^{11}$ in which several small superconductorinsulator-normal metal $(S I N)$ junctions are attached to a mesoscopic superconductor to detect small changes in the local density of states caused by supercurrents. By taking into account the axial symmetry of the disk, we were able to distinguish directly between MVSs and GVSs in a thin mesoscopic superconducting disk. ${ }^{12}$ Moreover, the experimental results were in very good agreement with the theoretical predictions from the nonlinear Ginzurg-Landau theory.
Subsequently, we found a less direct method to get information about GVSs and MVSs in mesoscopic superconducting disks. ${ }^{13}$ We showed that by studying the temperature dependence of the expulsion fields, one can distinguish between MVSs and GVSs. When the superconductor is in the MVS just before one vortex is expelled, we found that the expulsion field is almost independent of temperature. In fact, it decreases slightly. When, on the other hand, the last vortex state is a GVS, the expulsion field increases with increasing temperature.

Recently, nonaxially symmetric samples such as squares and triangles were investigated. Theoretical studies ${ }^{14-19}$ showed that different types of vortex states can appear in such samples: giant vortex states, multivortex states, combinations of giant and multivortex states, and even states where vortices and antivortices coexist. ${ }^{14}$ In addition, it became clear that the stability of the different vortex states strongly depends on the sample shape. Experimentally, both resistivity measurements ${ }^{7,20}$ and Hall magnetometry ${ }^{21}$ have been performed on mesoscopic superconducting squares and triangles. $H-T$ phase diagrams were constructed and the stability of the different vortex states were studied.

Very recently, Grigorieva et al. ${ }^{22}$ succeeded in the direct observation of vortex shells in mesoscopic superconducting $\mathrm{Nb}$ disks using the Bitter decoration technique. At fixed temperature, they studied well-defined shell structures containing up to 40 vortices and identified rules of shell filling and magic numbers, in agreement with Ref. 6. Also squares and triangles were studied but no evidence for giant vortex states was found.

In the present paper, we theoretically and experimentally investigate the different types of vortex states in nonaxially symmetric samples, placing an emphasis on whether (abovementioned) two methods, i.e., the MSTJ method and the temperature dependence of the vortex expulsion fields, are still able to distinguish between MVSs and GVSs for such nonaxially symmetric samples. Here we limit ourselves to super- 
(a)
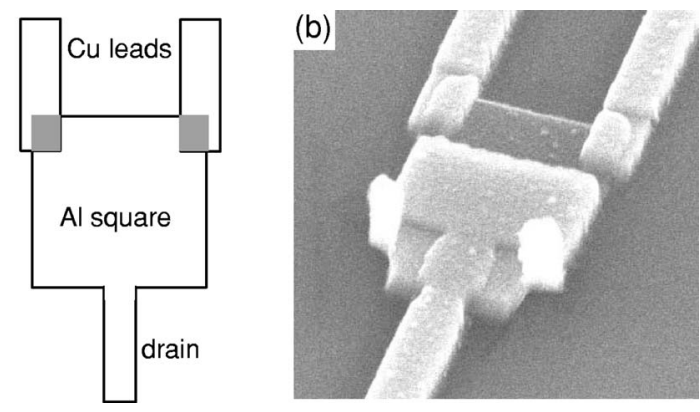

FIG. 1. Schematic view (a) and scanning electron micrograph (b) of a square sample with side $W=0.87 \mu \mathrm{m}$, fabricated using $e$-beam lithography followed by double-angle evaporation of $\mathrm{Al}$ and $\mathrm{Cu}$. Two small tunnel junctions (shaded regions) are placed on adjacent corners of the $\mathrm{Al}$ square which is directly connected to a drain lead. After the Al film was deposited, the surface of the Al film was slightly oxidized to provide the tunnel barrier. A part of the $\mathrm{Al}$ square is covered with a $\mathrm{Cu}$ film (bright regions). We expect that the $\mathrm{Cu}$ film will not have any serious influence on the superconductivity of the $\mathrm{Al}$ square because of the insulating $\mathrm{AlO}_{x}$ layer between them.

conducting squares. We also discuss the dependence of the MVSs and GVSs on sample size. Experimental results are compared with the theoretical prediction within the framework of the nonlinear Ginzburg-Landau theory.

The paper is organized as follows. In Sec. II we describe the experimental setup. In Sec. III we present the theoretical formalism to obtain the vortex states in thin mesoscopic superconductors. Next, we discuss the validity of the MSTJ method to distinguish directly between MVSs and GVSs when the superconducting sample is not axially symmetric (Sec. IV). In Sec. V we experimentally and theoretically study the temperature dependence of the transition fields. We also discuss the influence of the sample size. Next, in Sec. VI, we discuss the temperature dependence of the stability of the different vortex states, by considering the magnetic field region over which the vortex states are (meta)stable. Finally, in Sec. VII we summarize our results.

\section{EXPERIMENTAL SETUP}

We fabricated mesoscopic superconducting squares with small SIN junctions for detection of a change in the local supercurrent density and measured the vortex penetration and expulsion fields as a function of temperature. Figure 1 shows a schematic drawing and a scanning electron micrograph of a sample with side $W=0.87 \mu \mathrm{m}$. Two normal-metal (Cu) leads are connected to adjacent corners of a superconducting Al square through highly resistive small SIN tunnel junctions with nominal resistance around $0.5 \mathrm{M} \Omega$. Squares with five different sides $W=0.40,0.50,0.71,0.87$, and $1.0 \mu \mathrm{m}$ were fabricated. The coherence length $\xi(T=0)$ $=0.15-0.19 \mu \mathrm{m}$ was determined from the resistivity of the Al films deposited in the same way. These side dimensions correspond to $(2.1-2.7) \xi$, (2.6-3.3) $\xi$, (3.7-4.7) $\xi$, (4.6-5.8) $\xi$, and $(5.3-6.7) \xi$, respectively. The thickness of the $\mathrm{Al}$ squares and $\mathrm{Cu}$ leads are 33 and $65 \mathrm{~nm}$, respectively. The $\mathrm{Al}$ square is directly connected to a drain lead. The samples were fabricated using $e$-beam lithography followed by double-angle evaporation of $\mathrm{Al}$ and $\mathrm{Cu}$ in a chamber with a base pressure of $2 \times 10^{-8} \mathrm{~Pa}$. The superconducting transition temperature is $1.3-1.4 \mathrm{~K}$.

The samples were cooled in a dilution fridge that was equipped with lowpass noise filters for measurement lines in the lowest temperature part. In the measurement, we fixed the current flowing through one of the junctions to $100 \mathrm{pA}$, and measured the voltage between the $\mathrm{Cu}$ lead and the drain as a function of applied perpendicular magnetic field. The sweep rate was $5 \mathrm{mT} / \mathrm{min}$.

\section{THEORETICAL FORMALISM}

Theoretically, we follow the approach of Refs. 1 and 2 and solve numerically the two nonlinear Ginzburg-Landau equations self-consistently. Since $d \ll \xi, \lambda$, it is allowed to average the GL equations over the sample thickness. Using dimensionless variables and the London gauge $\operatorname{div} \vec{A}=0$ for the vector potential $\vec{A}$, we write the system of GL equations in the following form:

$$
\begin{gathered}
\left(-i \vec{\nabla}_{2 \mathrm{D}}-\vec{A}\right)^{2} \Psi=\Psi\left(1-|\Psi|^{2}\right), \\
-\Delta_{3 \mathrm{D}} \vec{A}=\frac{d}{\kappa^{2}} \delta(z) \vec{j}_{2 \mathrm{D}},
\end{gathered}
$$

where

$$
\vec{j}_{2 \mathrm{D}}=\frac{1}{2 i}\left(\Psi^{*} \vec{\nabla}_{2 \mathrm{D}} \Psi-\Psi \vec{\nabla}_{2 \mathrm{D}} \Psi^{*}\right)-|\Psi|^{2} \vec{A},
$$

is the density of superconducting current. The superconducting wave function satisfies the boundary conditions $\left(-i \vec{\nabla}_{2 \mathrm{D}}\right.$ $-\vec{A})\left.\Psi\right|_{n}=0$ normal to the sample surface and $\vec{A}=\frac{1}{2} H_{0} \rho \vec{e}_{\phi}$ far away from the superconductor. Here the distance is measured in units of the coherence length $\xi$, the vector potential in $c \hbar / 2 e \xi$, and the magnetic field in $H_{c 2}=c \hbar / 2 e \xi^{2}=\kappa \sqrt{2} H_{c}$. The superconductor is placed in the $(x, y)$ plane, the external magnetic field is directed along the $z$ axis, and the indices 2D, 3D refer to two- and three-dimensional operators, respectively.

By sweeping up and down the magnetic field we can find the different (meta)stable vortex states and their stability range. By comparing the dimensionless Gibbs free energies of the different vortex configurations

$$
F=V^{-1} \int_{V}\left[2\left(\vec{A}-\vec{A}_{0}\right) \cdot \vec{j}_{2 \mathrm{D}}-|\Psi|^{4}\right] d \vec{r},
$$

where integration is performed over the sample volume $V$ and $\vec{A}_{0}$ is the vector potential of the uniform applied magnetic field we find the ground state.

The temperature is indirectly included in $\xi, \lambda, H_{c 2}$, whose temperature dependence is given by

$$
\xi(T)=\frac{\xi(0)}{\sqrt{\left|1-T / T_{c 0}\right|}},
$$




$$
\begin{gathered}
\lambda(T)=\frac{\lambda(0)}{\sqrt{\left|1-T / T_{c 0}\right|}}, \\
H_{c 2}(T)=H_{c 2}(0)\left|1-\frac{T}{T_{c 0}}\right|,
\end{gathered}
$$

where $T_{c 0}$ is the critical temperature at zero magnetic field. Notice further that the Ginzburg-Landau parameter $\kappa=\lambda / \xi$ is independent of the temperature.

\section{MSTJ METHOD}

In the case of a mesoscopic superconducting disk we could experimentally distinguish between MVSs and GVSs using the MSTJ method. ${ }^{12}$ For a disk, the GVS has the same Cooper-pair density and the same superconducting current density everywhere along the boundary, while this is not so for the pinned MVS. So, if one places detectors (small tunnel junctions) at the boundary, one can distinguish these vortex states by comparing the voltage over those junctions; if the voltages take the same value, the vortex state is a GVS, and otherwise, a MVS.

For squares the situation is more complicated. Now, even for a GVS the current at the boundary depends on the exact position; the Cooper-pair density and the supercurrent distribution of a GVS do not have axial symmetry, but only tetragonal symmetry. Therefore, it is important to attach the tunnel junctions to symmetrical positions at the sample boundary, as shown in Fig. 1, where two tunnel junctions are placed on adjacent corners. But, even with symmetrical junctions, it is in most cases not possible to distinguish between MVSs and GVSs. The reason is that most multivortex configurations have tetragonal or mirror symmetry. This can be seen from Fig. 2 where we show some examples of multivortex and giant vortex states in a mesoscopic square, as calculated within the framework of the nonlinear GinzburgLandau theory. Figures 2(a)-2(f) show the Cooper-pair density of the multivortex states with vorticity $L=3,4,5,6,8$, and 10 for a square with width $W=10 \xi$ and thickness $d$ $=0.1 \xi$ at $T=0.4 \mathrm{~K}$. Notice that $\xi$ is the coherence length at $T=0 \mathrm{~K}$. For multivortex states as shown in Figs. 2(b)-2(e), junctions connected to the symmetrical positions as shown in Fig. 1 will not lead to different voltages and one could wrongly think that the states are giant vortex states in the MSTJ measurement. This problem always occurs for samples without axial symmetry.

\section{TEMPERATURE DEPENDENCE OF VORTEX TRANSITION FIELDS}

In Ref. 13 we showed how the temperature dependence of the expulsion field gives us information on the type of vortex state which is nucleated in the superconducting disk. When the vortex state is a MVS just before one vortex is expelled, then the expulsion fields are almost independent (in fact, they decrease very slightly) of temperature. When, on the other hand, the last vortex state is a GVS, then the expulsion fields increase with increasing temperature. Thus, the temperature dependence of the transition fields can indirectly

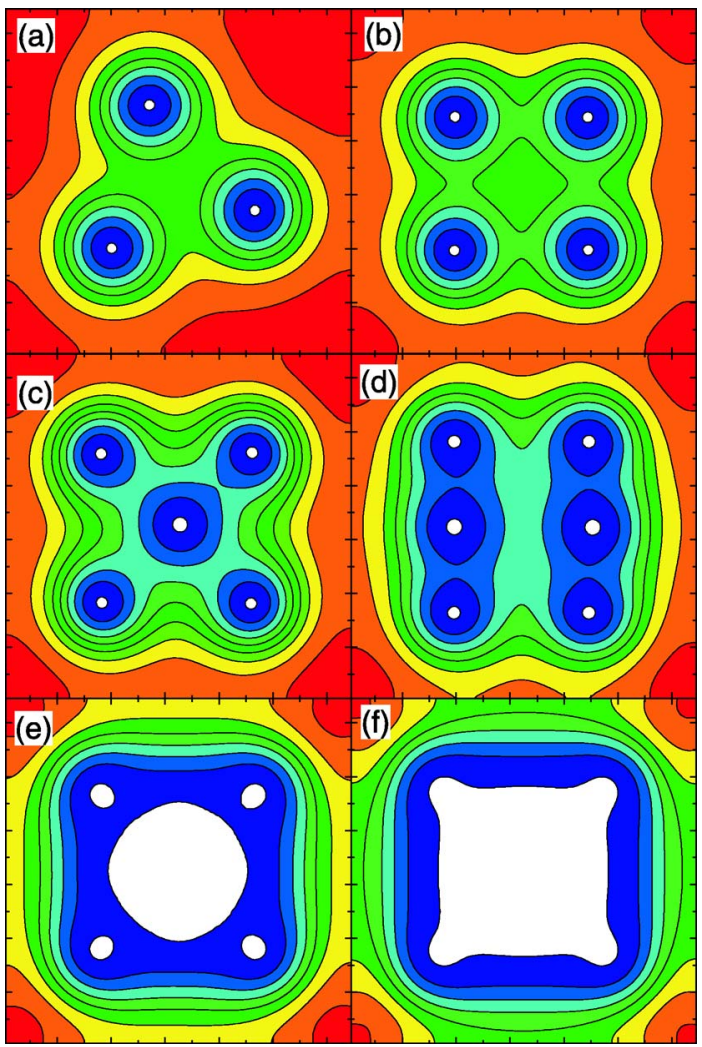

FIG. 2. (Color online) The Cooper-pair density for (a) the $L$ $=3$ state at $H=0.305 H_{c 2}(T=0), \quad($ b) the $L=4$ state at $H$ $=0.385 H_{c 2}(T=0)$, (c) the $L=5$ state at $H=0.465 H_{c 2}(T=0)$, (d) the $L=6$ state at $H=0.525 H_{c 2}(T=0)$, (e) the $L=8$ state at $H$ $=0.690 H_{c 2}(T=0)$, and (f) the $L=10$ state at $H=0.865 H_{c 2}(T=0)$, in a square with width $W=10 \xi$ and thickness $d=0.1 \xi$. The temperature is $T=0.4 \mathrm{~K}$. Each figure shows the vortex configuration just before vortex expulsion. High (low) Cooper-pair density is indicated by red (blue) regions. (a)-(d) show the multivortex states, (e) a combination of a multivortex state and a giant vortex state, and (f) the giant vortex state.

distinguish between MVSs and GVSs. Since the transition fields can be easily obtained through resistivity, tunnel, or magnetization measurements, this method might become a powerful tool to determine the type of vortex states.

\section{A. Experimental results}

Experimentally, we measure the junction voltage under a bias current of $100 \mathrm{pA}$ as a function of applied magnetic field for squares with different sizes and at different temperatures. Figure 3 shows, as an example, the measured voltage for a square with sides $W=0.87 \mu \mathrm{m}$ for increasing [Fig. 3(a)] and decreasing applied field [Fig. 3(b)] at different values of the temperature. The highest curve corresponds to the lowest temperature and the voltage decreases when the temperature increases. The voltage variation as a function of magnetic field results from two origins: (1) smearing of the superconducting energy gap due to pair breaking by the magnetic field and (2) a decrease of the energy gap because of the supercurrent, i.e., the Meissner effect. In particular, the jumps indicate the fields where one vortex enters or leaves the 


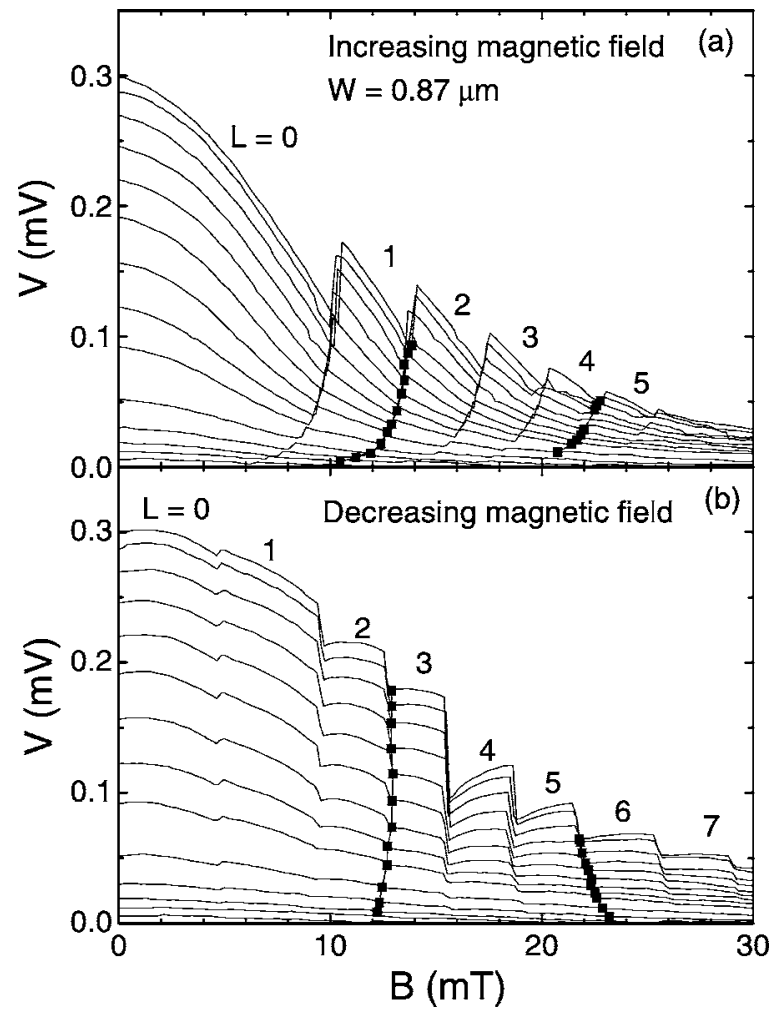

FIG. 3. The measured voltage as a function of (a) increasing magnetic field and (b) decreasing magnetic field for a square with sides $W=0.87 \mu \mathrm{m}$ at different temperatures, i.e., $T=0.15$ (highest curve), $0.20,0.25,0.30,0.35,0.40,0.45,0.50,0.55,0.65,0.75$, $0.85,0.95$, and $1.05 \mathrm{~K}$ (lowest curve). The square symbols in (a) indicate the $L=1 \rightarrow 2$ and the $L=4 \rightarrow 5$ transition fields, and in (b) the $L=3 \rightarrow 2$ and the $L=6 \rightarrow 5$ transition fields.

sample, i.e., the penetration or expulsion fields. From Fig. 3 (a) it can be seen that the $L \rightarrow L+1$ penetration fields decrease with increasing temperature. To illustrate this more clearly we mark the peaks corresponding to the $L=1 \rightarrow 2$ and the $L=4 \rightarrow 5$ transition fields by black symbols in the figure. With decreasing field, we find again two regimes, as we found in the case of a disk (see Ref. 13). At low fields the expulsion fields slightly decrease with temperature. See, for example, the black symbols that indicate the $L=3 \rightarrow 2$ expulsion fields. At higher fields, on the other hand, the expulsion fields increase with temperature. This can be seen clearly from the black symbols indicating the $L=6 \rightarrow 5$ transition.

We repeated this measurement for several square samples with different sizes, i.e., $W=0.40,0.50,0.71,0.87,1.0 \mu \mathrm{m}$, and we plot the resulting transition fields as a function of temperature in Fig. 4. In Figs. 4(a)-4(e) we increase the applied magnetic field and the penetration fields are given, while in Figs. 4(f)-4(j) we decrease the applied magnetic field and the expulsion fields are shown. ${ }^{24}$

For all sample sizes, we find that the penetration fields decrease almost uniformly with increasing temperature [Figs. 4(a)-4(e)]. This result is analogous to the results that we found in the case of a disk. ${ }^{13}$ With increasing sample size, more vortex states become stable and for fixed $L$ the $L \rightarrow L$ +1 penetration moves to lower fields.

With decreasing field, we find that for $W=0.40 \mu \mathrm{m}$ the expulsion fields always increase with increasing temperature [see Fig. 4(f)]. If our method for distinction between MVS and GVS is still valid for nonaxially symmetric samples, this would mean that we only find GVSs in small samples. For larger squares, we find again two regimes just as we found for a disk. At high fields the $L \rightarrow L-1$ expulsion fields increase with temperature, but at low fields they are constant or even decrease slightly over a certain temperature region before they start to increase again. For $W=0.50 \mu \mathrm{m}$, expulsion fields are constant at low temperatures for $L=2$ [see Fig. 4(g)], for $W=0.71 \mu \mathrm{m}$ for $L=2,3,4$ [see Fig. 4(h)], for $W$ $=0.87 \mu \mathrm{m}$ for $L=2-5$ [see Fig. 4(i)], and for $W=1.0 \mu \mathrm{m}$ for $L=2-6$ [see Fig. 4(h)], corresponding to the MVSs before the transition (if the method is still valid). Assuming that our method is indeed still valid, the figure would indicate that the MVS becomes more stable when the sample size increases.

Notice that in Figs. 4(g) $-4(\mathrm{j})$ the $L=4$ curve is shifted towards lower magnetic field, which leads to a smaller distance in magnetic field between $L=3$ and $L=4$ and a larger distance between $L=4$ and $L=5$. This is a clear manifestation of a commensurability effect, ${ }^{17}$ indicating that four vortices can more easily be accommodated in a square sample than 3 or 5 vortices.

\section{B. Theoretical results}

Now, we theoretically check whether such an indirect distinction is valid for nonaxially symmetric superconductors. We calculate the free energy as a function of applied magnetic field for different sizes of the square sample and for different temperatures. As an example, we show in Fig. 5 the free energy for the vortex states in a square with width $W$ $=10 \xi$ and thickness $d=0.1 \xi$ when sweeping up [Fig. 5(a)] and when sweeping down [Fig. 5(b)] the applied magnetic field for different values of the temperature, i.e., $T=0.1 \mathrm{~K}$ (lowest curve) to $0.8 \mathrm{~K}$ (highest curve) with steps of $0.1 \mathrm{~K}$. Jumps in the free energy correspond to the penetration or the expulsion of a vortex with vorticity change of \pm 1 . When increasing the magnetic field, we find that the transition fields always decrease with temperature, just as in the case of a circular disk. ${ }^{13}$ When decreasing the magnetic field, we find again two regimes; the expulsion fields are almost temperature independent at low fields, while at higher fields they increase with temperature. To show this clearly we mark several transitions by solid black square symbols in the figure.

We calculated the same for squares with sides $W=5 \xi$ and $8 \xi$. In Figs. 6(a)-6(c) we show the penetration fields and in Figs. 6(d)-6(f) the expulsion fields as a function of temperature for squares with $W=5 \xi(\mathrm{a}),(\mathrm{d}), 8 \xi(\mathrm{b}),(\mathrm{e})$, and $10 \xi(\mathrm{c})$, (f). We find that for all samples the penetration fields decrease uniformly with increasing temperature, just as we found experimentally. The behavior of the expulsion fields is more interesting. With decreasing field, we find for the smallest sample, i.e., the square with $W=5 \xi$ [see Fig. 6(d)], that the $L \rightarrow L-1$ expulsion fields increase with temperature for all values of the vorticity $L$. For larger samples we find again that the expulsion fields are almost temperature independent at low fields and increase at higher fields. In the case of the disk we know that the behavior depends on the fact whether the last vortex state is a MVS or a GVS. To inves- 


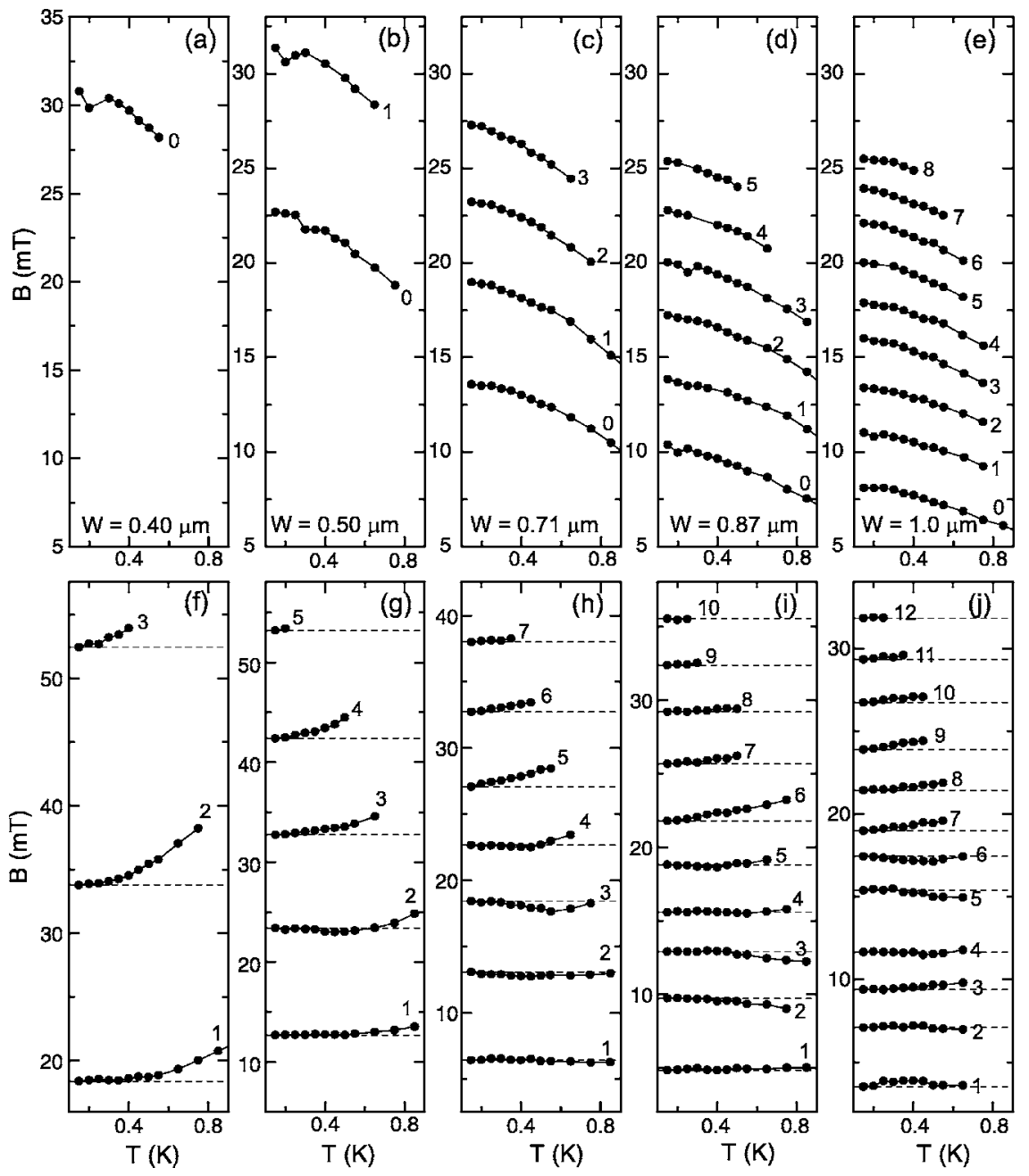

FIG. 4. (a)-(e) The experimental $L \rightarrow L+1$ penetration and (f)(j) $L \rightarrow L-1$ expulsion fields as a function of temperature for square samples with different width $W$, i.e., $W=0.40 \mu \mathrm{m}$ (a), (f), $0.50 \mu \mathrm{m}$ (b), (g), $0.71 \mu \mathrm{m} \quad$ (c), (h), $0.87 \mu \mathrm{m}(\mathrm{d}),(\mathrm{i})$, and $1.0 \mu \mathrm{m}$ (e), (j). These sides correspond to $(2.1-2.7) \xi(\mathrm{a}),(\mathrm{f}),(2.6-3.3) \xi(\mathrm{b})$,

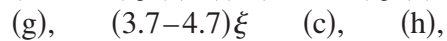
$(4.6-5.8) \xi(\mathrm{d}),(\mathrm{i})$, and $(5.3-6.7) \xi$ (e), (j), respectively. The indices indicate the vorticity $L$. The dashed lines correspond to the values of the expulsion field at the lowest temperature and are guides to the eye.

tigate whether this is still valid for squares, we checked the Cooper-pair density of the vortex states, just before the transition. Some examples are given in Fig. 2. When the last state is a GVS, the transition field is given by an open symbol in Fig. 6 and when it is a MVS by a closed symbol. For $W=5 \xi$ we find only GVSs. Here, all vortices are compressed in the sample center, because the sample is too small and the influence of the boundary is too strong. Notice that we found similar experimental results for even smaller samples. The behavior of the expulsion fields as a function of temperature is in good agreement with the prediction for a disk and with the experimental results for squares with $W=0.4 \mu \mathrm{m}$ [see Fig.4(f))]. When we increase the sample size to $W=8 \xi$ and $10 \xi$, MVSs can stabilize in certain magnetic field regions, as are indicated in Figs. 6(e) and 6(f) by the black symbols. Notice that the number of vorticities that have MVSs increases with the sample size; For $W=8 \xi$ MVSs appear in $L$ $=2$ to 6 , while for $W=10 \xi$ in $L=2$ to 11 at $T=0.1 \mathrm{~K}$. This is also in good qualitative agreement with the experimental results.

In Figs. 6(d)-6(f), we generally see that the expulsion fields are almost temperature independent when the state is a MVS, and they increase when the state is a GVS, as in the case of a disk. However, the situation is less clear than in the case of a disk. Consider, for example, the $L=8 \rightarrow 7$ transition field for $W=10 \xi$ as a function of temperature. At low tem- peratures, the expulsion field is temperature independent. At $T=0.4 \mathrm{~K}$ the expulsion field begins to increase, although the state is still a MVS. Then, for $T \geqslant 0.5 \mathrm{~K}$ the state is a GVS and the expulsion field increases as expected. Below we study the $L=8$ state at $T=0.4 \mathrm{~K}$ in more detail. Although the vortex state just before the vortex expulsion is a MVS according to our criterion, ${ }^{23}$ the Cooper-pair density approaches the GVS configuration (see below). The reason is that the maximum Cooper-pair density between two separated vortices is only of the order of $10^{-2}$ (normalized by the value at $B=0$ ). Thus, the Cooper-pair density and also the supercurrent distribution near the boundary are very close to those for a GVS [see, e.g., Figs. 2(e) and 2(f)]. In spite of these kinds of uncertainties for some vorticities, we believe that in general one can still distinguish MVSs and GVSs by studying the temperature dependence of the expulsion fields, even though the sample is not axially symmetric anymore.

At the transition fields the vortex state is either a MVS or a GVS. We do not find a combination of the two vortex states near the expulsion or penetration fields for the considered situations. However, from, e.g., Fig. 2(e) and Ref. 17 we know that such a combination can stabilize away from the transition fields. This is illustrated in Fig. 7 for the $L=8$ state in a square with $W=10 \xi$ at $T=0.4 K$, where we show the free energy of all the (meta)stable vortex states with $L=8$. The insets show the Cooper-pair density at the fields indicated by 


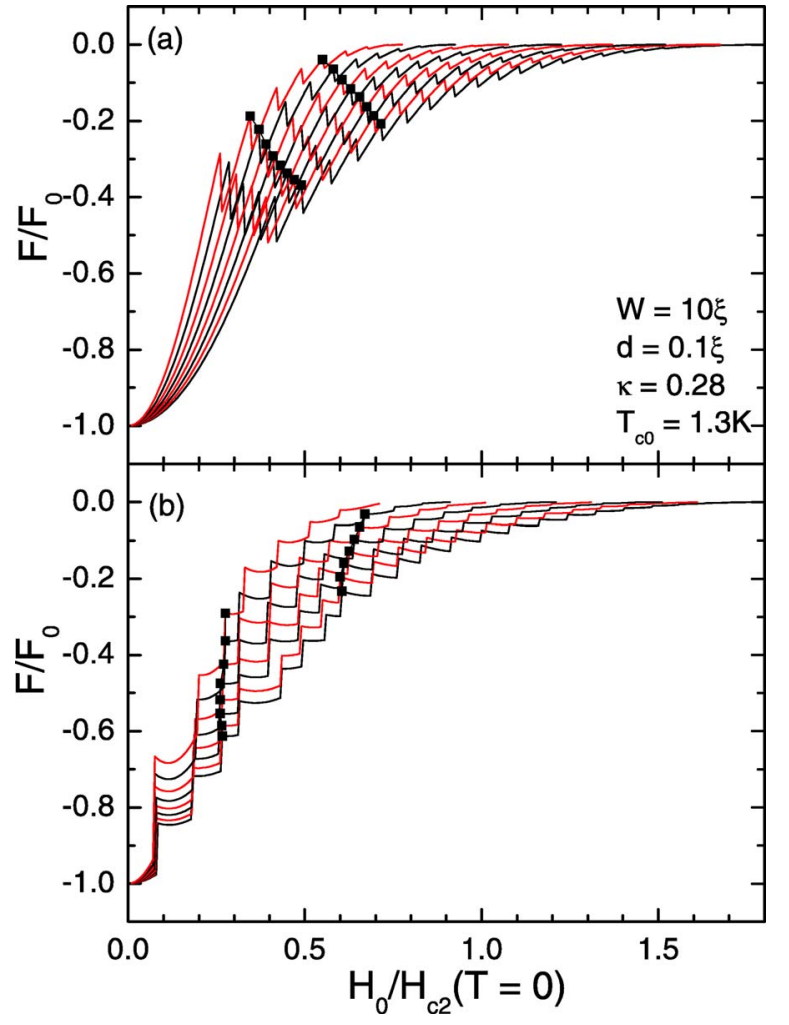

FIG. 5. (Color online) The free energy as a function of applied magnetic field for the vortex states in a square with $W=10 \xi, d$ $=0.1 \xi$ for different values of the temperature for (a) increasing magnetic field and (b) decreasing magnetic field. The temperatures are $T=0.1$ (lowest curve), 0.2, 0.3, 0.4, 0.5, 0.6, 0.7, and $0.8 \mathrm{~K}$ (highest curve). The square symbols in (a) indicate the $L=1 \rightarrow 2$ and the $L=4 \rightarrow 5$ transition fields, and in (b) the $L=2 \rightarrow 1$ and the $L=8$ $\rightarrow 7$ transition fields.

the arrows. At low fields $\left[H_{0}<0.665 H_{c 2}(T=0)\right]$ we find eight separated vortices, i.e., four vortices towards the corners and four vortices towards the middle of the sides [see inset (a)]. However, with increasing field the four vortices which are situated near the middle of the sides move towards the center of the square and at $H_{0}=0.665 H_{c 2}(T=0)$ they merge and form a giant vortex with $L=4$ in the center, which coexist with four single vortices localized near the corners [see inset (b)]. The vortex state is now a combination of a GVS and a MVS. With increasing field the four single vortices move towards the central vortex and at $H_{0}$ $=0.745 H_{c 2}(T=0)$ the four single vortices merge with the giant vortex and a nonaxial symmetric GVS with $L=8$ stabilizes [see inset (c)].

When comparing the theoretical and experimental results (Figs. 6 and 4) in more detail, one notices that the experimental samples favor MVSs strongly in comparison with the theoretical ones; for example, experimental squares with sides $W=0.71 \mu \mathrm{m}$ [corresponding to $(2.6-3.3) \xi$ ], and $0.87 \mu \mathrm{m}[(3.7-4.7) \xi]$ have MVSs for certain vorticity ranges, while theoretically a bigger sample with $W=5 \xi$ does not have a MVS region. One possible reason for this discrepancy is the thickness of the sample. The thickness of the experimental sample $d=33 \mathrm{~nm}$ is larger than the thickness

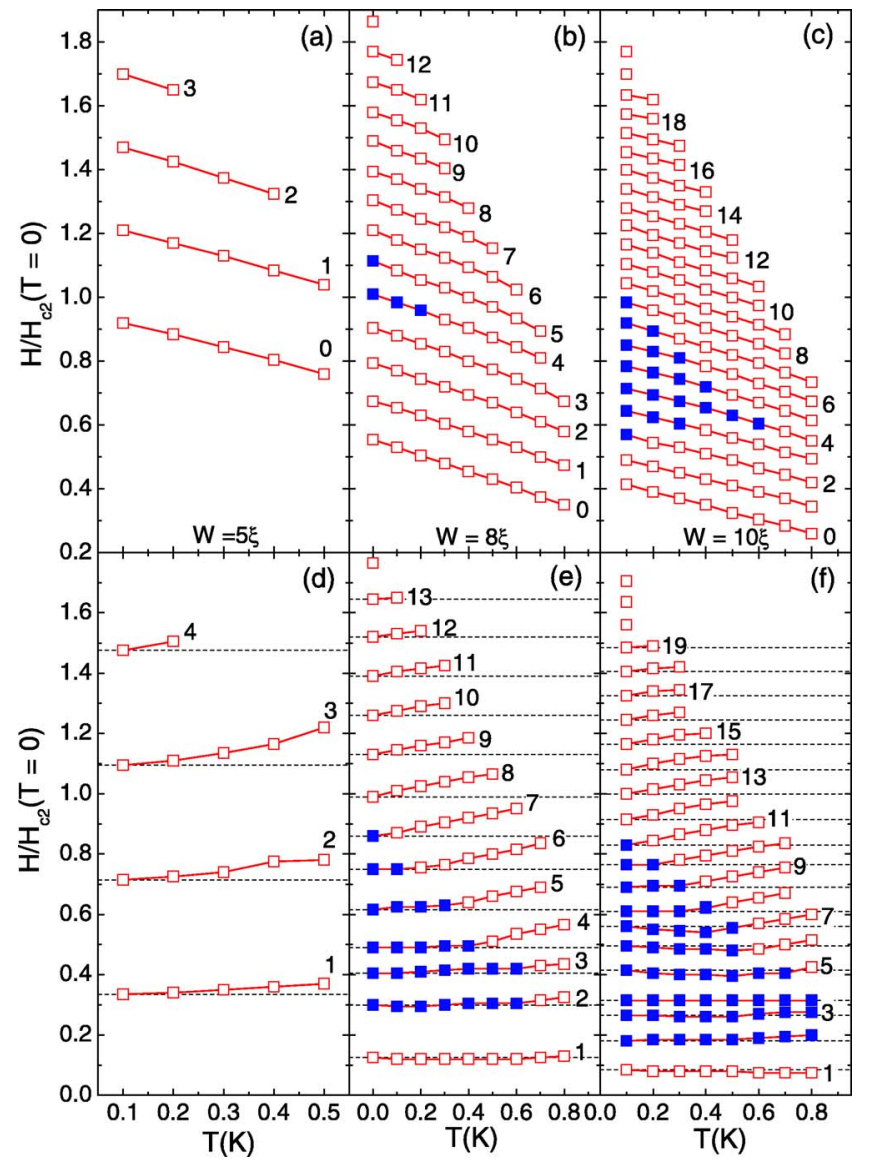

FIG. 6. (Color online) (a)-(c) The calculated $L \rightarrow L+1$ penetration and (d)-(f) $L \rightarrow L-1$ expulsion fields as a function of temperature for square samples with different width $W$, i.e., $W=5 \xi(\mathrm{a}),(\mathrm{d})$, $8 \xi$ (b), (e), and $10 \xi$ (c), (f). The sample thickness is always $d$ $=0.1 \xi$ and $\kappa=0.28$. The indices indicate the vorticity $L$. Closed (blue) symbols correspond to a MVS and open (red) symbols to a GVS just before the transition. The dashed lines correspond to the values of the expulsion field at the lowest temperature and are guides to the eye. The critical temperate is taken to be $T_{c 0}=1.3 \mathrm{~K}$.

used in the theoretical simulation $(0.1 \xi)$. But we do not think this is the major reason. Our previous study reveals that the effective thickness in experimental samples becomes smaller than the actual thickness, presumably due to surface roughness and the oxide layer, and the effective thickness for a $33 \mathrm{~nm} \mathrm{Al} \mathrm{film} \mathrm{is} \mathrm{close} \mathrm{to} 0.1 \xi^{12}$ Also it is known that the phase boundary of the vortex states is less sensitive to the thickness of the samples. ${ }^{2}$ Another uncertainty is the expression for the temperature dependence of $\lambda$ and $\xi$. Equations (5)-(7) are only valid near $T_{c}$. At low temperatures one should use the expressions as obtained within a two-fluid approximation, ${ }^{25,26}$ where the penetration depth varies as

$$
\lambda(T)=\lambda(0) \frac{1}{\sqrt{1-\left(T / T_{c 0}\right)^{4}}},
$$

and the coherence length as 


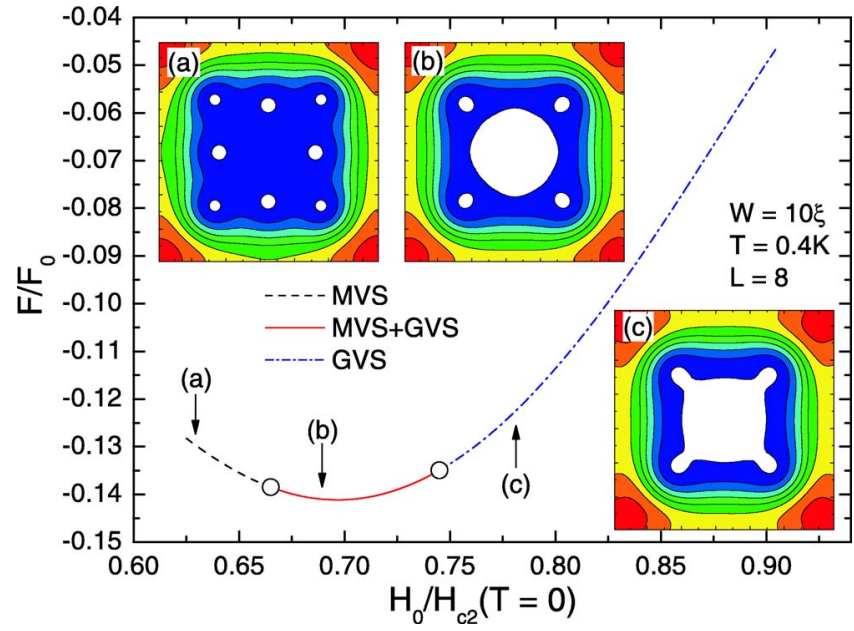

FIG. 7. (Color online) The free energy as a function of the applied magnetic field for all (meta)stable vortex states with $L=8$ in a square with $W=10 \xi$ at $T=0.4 \mathrm{~K}$, i.e., a MVS with eight single vortices (dashed curve), a combination of a $L=4 \mathrm{GVS}$ in the center with four separated vortices towards the corners (solid curve), and the $L=8$ GVS (dash-dotted curve). The open symbols indicate the transitions between the different configurations. The insets (a)-(c) show the Cooper-pair density of a MVS at $H_{0} / H_{c 2}(T=0)=0.63$, a combination of a MVS and a GVS at $H_{0} / H_{c 2}(T=0)=0.69$ and a GVS at $H_{0} / H_{c 2}(T=0)=0.78$. These fields are indicated by the arrows in the main figure. High (low) Cooper-pair density is given by red (blue) regions. White regions indicate the vortex cores with $|\Psi|^{2}<0.01$ (Ref. 23).

$$
\xi(T)=\xi(0) \frac{\sqrt{1+\left(T / T_{c 0}\right)^{2}}}{\sqrt{1-\left(T / T_{c 0}\right)^{2}}} .
$$

Notice that this leads to a temperature dependent $\kappa=\lambda / \xi$. At present there does not exist a reliable formula which interpolates the expressions for $\xi(T)$ and $\lambda(T)$ from the $T \rightarrow 0$ region to the $T \rightarrow T_{c}$ region. Although different expressions for the $T$ dependence of $\xi(T)$ and $\lambda(T)$ do not change the qualitative behavior of the theoretical results, they will result in quantitative difference in, e.g., the value of the expulsion and penetration fields. Another more perturbing effect will be the presence of possible defects inside the sample. Defects tend to localize vortices at the defect positions, so that multiple defects may make the MVSs more stable. Actually, defects can sometimes play a critical role when determining experimentally the type of the vortex state. ${ }^{12,27}$ A systematic study on the effect of defects is needed, but is beyond the scope of the present work. A simple criterion for the behavior of the penetration and expulsion fields is discussed in the Appendix.

\section{STABILITY OF THE DIFFERENT VORTEX STATES}

Next, we investigate the stability of the different vortex states in a superconducting square for different values of the temperature by studying the magnetic field region over which the different vortex states are (meta)stable. In Ref. 17 we studied this stability region at $T=0$ for a disk, a square and a triangle with the same surface area $S=16 \pi \xi^{2}(0)$. We

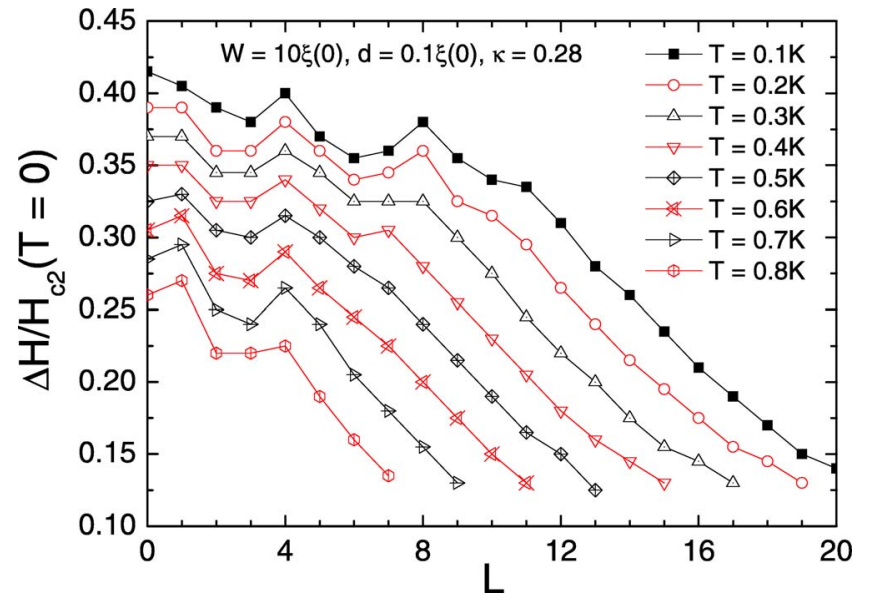

FIG. 8. (Color online) The theoretical magnetic field range over which the vortex states with vorticity $L$ are stable as a function of the vorticity $L$ for different temperature values.

found that for the disk this stability region uniformly decreases with increasing vorticity, while for the square we found a peak at $L=4$, indicating that this state has enhanced stability. For the triangle a peak occurs for $L=3$.

Experimentally, Morelle et al. ${ }^{21}$ performed Hall magnetometry measurements on a $\mathrm{Al}$ square and triangle and discussed the stability of the different vortex patterns at two temperatures close to $T_{c}$. But no systematic study of the temperature dependence of the vortex state stability was presented. In Ref. 21 peaks were found at $L=4$ in a square and one at $L=3$ in a triangle.

Now, we will study the temperature dependence of this enhanced stability at $L=4$ in a square sample with $W=10 \xi$ in more detail. Figure 8 shows the theoretically obtained magnetic field region over which the different vortex states are (meta)stable as a function of the vorticity $L$ for different temperature values. Since the sample is larger than the one considered in Ref. 17 we find at low temperatures not only a peak at $L=4$, but also at $L=8$. With increasing temperature, the effective size of the sample, i.e. $W / \xi(T)$, becomes smaller, less vortex states stabilize and the peak at $L=8$ disappears, leading us to the situation of Ref. 17.

To study how much the stability is enhanced at $L=4$ as compared to $L=3$ and $L=5$ we linearly interpolate between the values at $L=3$ and $L=5$, and then we subtract the interpolated value for $L=4$ from the value of the peak at $L=4$. This result is shown in Fig. 9(a). We find that the temperature dependence of the stability enhancement at $L=4$ is a nonuniform function of the temperature with a clear peak near $T$ $=0.6-0.7 \mathrm{~K}$. Next, we repeat this for the experimental data for a square with $W=1.0 \mu \mathrm{m}$ and plot the result in Fig. 9(b). Also experimentally we found such a clear peak structure at $T=0.5-0.6 \mathrm{~K}$, which is slightly shifted to lower temperature as compared to the theoretical results. The enhancement is probably due to the fact that the size of the sample decreases compared to $\xi(T)$ [i.e., $W / \xi(T=0.6)=7.3$ and $W / \xi(T=0.7)$ $=6.8 \mathrm{~J}$ and this enhances the influence of the sample boundary on the multivortex state. From Fig. 6(f) we know that at $T=0.6 \mathrm{~K}$ and $0.7 \mathrm{~K}$, the state for $L=3$ and $L=5$ are still multivortex states. Since the sample boundary favors square 

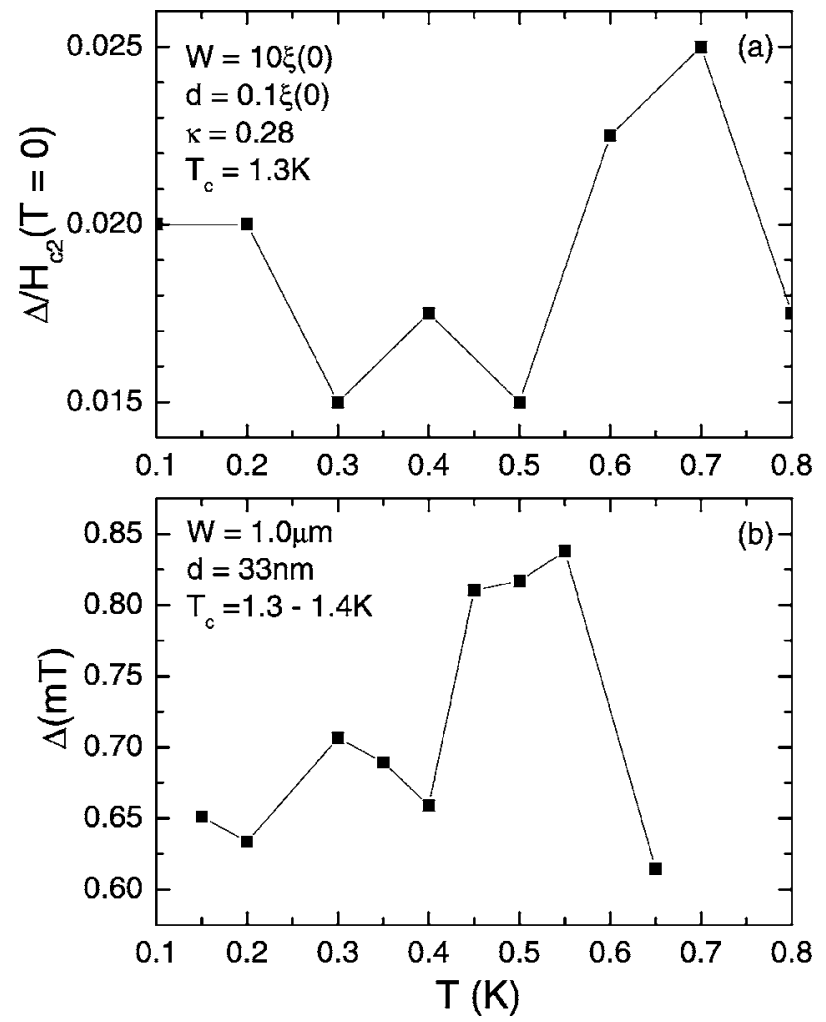

FIG. 9. Stability enhancement at $L=4$ as a function of temperature, (a) as obtained from the theoretical data and compared with (b) the experimental data for the sample parameters as indicated in the figure.

symmetry, the smaller stability of the $L=3$ and $L=5$ compared to the $L=4$ state becomes even more pronounced at these temperatures. At $T=0.8 \mathrm{~K}$, the $L=5$ state is a GVS which fits better in the square sample. Therefore, the peak at $L=4$ decreases a little bit (but it is still a peak). Regardless of a different effective sample size, the possible presence of defects, uncertainties about the sample thickness, the size of the coherence length and the value of the critical temperature, we can conclude that there is qualitative agreement between theory and experiment. The small shift in temperature can be explained by the fact that Eqs. (5)-(7) are only valid near the phase boundary (see above) and the experimental uncertainty on the value of the critical temperature. Notice that both experimentally $(T \sim 0.3 \mathrm{~K})$ and theoretically $(T$ $\sim 0.4 \mathrm{~K})$ an additional smaller peak structure is found at lower temperatures.

\section{CONCLUSIONS}

We investigated vortex states in mesoscopic superconducting squares, both experimentally by using the SIN tunnel junctions and theoretically within the framework of the nonlinear Ginzburg-Landau theory. First we tried to distinguish experimentally between multivortex and giant vortex states using the MSTJ method, but we encountered major difficulties concerning the importance of the location of the leads, due to the non-axial symmetry of the square sample. Then, we investigated the transition fields as a function of the tem- perature for square samples of different sizes. We found that the temperature behavior is similar to the case of disks, but the temperature dependence is less pronounced. With increasing field, the penetration fields decrease as a function of temperature, regardless of the type of vortex state. On the other hand, with decreasing field we find two regimes depending on the type of the vortex state. When the state is a multivortex state the expulsion fields are almost temperature independent. In the case of a giant vortex state they increase with increasing temperature. Using the temperature dependence of the vortex expulsion field, we experimentally verified that the multivortex states become more stable when the sample size increases. Finally, we investigated the temperature dependence of the stability of the different vortex states. The theoretical results are in qualitative agreement with the experimental data, even for the temperature dependence of the stability field of the $L=4$ vortex state.

\section{ACKNOWLEDGMENTS}

This work was supported by the University of Tsukuba Nanoscience Special Project, Grant-in-Aid for Scientific Research (B) (17340101) and CTC-NES Program of JSPS, the Flemish Science Foundation (FWO-Vl), the Belgian Science policy, and the ESF-AQDJJ program. B. J. Baelus acknowledges support from FWO-Vl. Useful discussions with D. Vodolazov are acknowledged.

\section{APPENDIX}

In this Appendix we give a simple estimate of the temperature dependence of the penetration and expulsion fields. The transition from one vortex state to the next one occurs when the supercurrent at a certain point near the edge of the sample is sufficiently large. Is this case the barrier for vortex entry or vortex exit can be overcome. For a giant vortex with vorticity $L$ in a small sample the criterion for vortex transition can be obtained if we consider the component of the supercurrent parallel to the sample boundary, i.e.,

$$
\left|\left(\frac{2 e A}{\hbar c}-\nabla \varphi\right)_{\tau}\right|=\left|\frac{e H_{0} R}{\hbar c}-\frac{L}{R}\right| \sim \frac{1}{\xi},
$$

where $\varphi$ is the phase of the order parameter and $R$ is the characteristic distance from the sample center to the edge. Notice that in this approach we assume a very thin sample such that demagnetization effects can be neglected.

We can obtain the following estimate for the penetration fields $H_{p}$ and expulsion fields $H_{e}$ :

$$
\begin{aligned}
& H_{p} \sim \frac{L \phi_{0}}{\pi R^{2}}+\frac{\phi_{0}}{\pi \xi R}=\frac{L \phi_{0}}{\pi R^{2}}+\frac{\phi_{0}}{\pi \xi_{0} R} \sqrt{1-\frac{T}{T_{c}}}, \\
& H_{e} \sim \frac{L \phi_{0}}{\pi R^{2}}-\frac{\phi_{0}}{\pi \xi R}=\frac{L \phi_{0}}{\pi R^{2}}-\frac{\phi_{0}}{\pi \xi_{0} R} \sqrt{1-\frac{T}{T_{c}}},
\end{aligned}
$$

where $\phi_{0}$ is the flux quantum. This simple estimate explains us why the penetration fields should decrease with increasing temperature and the expulsion fields should increase with 
increasing temperature. This estimate is expected to hold even when the giant vortex state splits into a vortex molecule (multivortex state), provided that the size of the vortex molecule is much smaller than $R$.

Next, we check whether the results of Fig. 6 agree with this simple criterion. We found that the penetration fields decrease with increasing temperature, both for multivortex and giant vortex states, as is predicted by the above criterion. On the other hand, we found that the expulsion fields are almost temperature independent when the state is a multivor- tex state, while they increase with increasing temperature in the case of a giant vortex state. Therefore, the criterion is still valid for giant vortex states, but not for multivortex states. The reason is that near the expulsion field the single vortices in a multivortex state are no more concentrated near the sample center but are located more towards the edge of the superconductor [see, e.g., Fig. 7(a)], and therefore the size of the vortex molecule is no longer much smaller than $R$. Notice that near the penetration fields, the single vortices of a multivortex state are close to the sample center.
*Email address: ben.baelus@ua.ac.be

†Email address: kanda@1t.px.tsukuba.ac.jp

†mail address: francois.peeters@ua.ac.be

${ }^{1}$ V. A. Schweigert and F. M. Peeters, Phys. Rev. B 57, 13817 (1998).

${ }^{2}$ V. A. Schweigert, F. M. Peeters, and P. S. Deo, Phys. Rev. Lett. 81, 2783 (1998).

${ }^{3}$ J. J. Palacios, Phys. Rev. B 58, R5948 (1998).

${ }^{4}$ J. J. Palacios, Phys. Rev. Lett. 84, 1796 (2000).

${ }^{5}$ B. J. Baelus, L. R. E. Cabral, and F. M. Peeters, Phys. Rev. B 69, 064506 (2004).

${ }^{6}$ L. R. E. Cabral, B. J. Baelus, and F. M. Peeters, Phys. Rev. B 70, 144523 (2004).

${ }^{7}$ V. V. Moshchalkov, L. Gielen, C. Strunk, R. Jonckheere, X. Qiu, C. Van Haesendonck, and Y. Bruynseraede, Nature (London) 373, 319 (1995).

${ }^{8}$ V. Bruyndoncx, L. Van Look, M. Verschuere, and V. V. Moshchalkov, Phys. Rev. B 60, 10468 (1999).

${ }^{9}$ A. K. Geim, I. V. Grigorieva, S. V. Dubonos, J. G. S. Lok, J. C. Maan, A. E. Filippov, and F. M. Peeters, Nature (London) 390, 256 (1997)

${ }^{10}$ A. K. Geim, S. V. Dubonos, I. V. Grigorieva, K. S. Novoselov, F. M. Peeters, and V. A. Schweigert, Nature (London) 407, 55 (2000)

${ }^{11}$ A. Kanda, M. C. Geisler, K. Ishibashi, Y. Aoyagi, and T. Sugano, in Quantum Coherence and Decoherence ISQM-Tokyo '98, edited by Y. A. Ono and K. Fujikawa (Elsevier, Amsterdam, 1999), p. 229; A. Kanda and Y. Ootuka, Microelectron. Eng. 63, 313 (2002); A. Kanda and Y. Ootuka, Physica C 404, 205 (2004).

${ }^{12}$ A. Kanda, B. J. Baelus, F. M. Peeters, K. Kadowaki, and Y. Ootuka, Phys. Rev. Lett. 93, 257002 (2004).

${ }^{13}$ B. J. Baelus, A. Kanda, F. M. Peeters, Y. Ootuka, and K. Kadowaki, Phys. Rev. B 71, 140502(R) (2005).
${ }^{14}$ L. F. Chibotaru, A. Ceulemans, V. Bruyndoncx, and V. V. Moshchalkov, Nature (London) 408, 833 (2000).

${ }^{15}$ L. F. Chibotaru, A. Ceulemans, V. Bruyndoncx, and V. V. Moshchalkov, Phys. Rev. Lett. 86, 1323 (2001).

${ }^{16}$ J. Bonča and V. V. Kabanov, Phys. Rev. B 65, 012509 (2001); T. Mertelj and V. V. Kabanov, ibid. 67, 134527 (2003).

${ }^{17}$ B. J. Baelus and F. M. Peeters, Phys. Rev. B 65, 104515 (2002).

${ }^{18}$ A. S. Mel'nikov, I. M. Nefedov, D. A. Ryzhov, I. A. Shereshevskii, V. M. Vinokur, and P. P. Vysheslavtsev, Phys. Rev. B 65, 140503(R) (2002).

${ }^{19}$ V. R. Misko, V. M. Fomin, J. T. Devreese, and V. V. Moshchalkov, Phys. Rev. Lett. 90, 147003 (2002).

${ }^{20}$ M. Morelle, G. Teniers, L. F. Chibotaru, A. Ceulemans, and V. V. Moshchalkov, Physica C 369, 351 (2002).

${ }^{21}$ M. Morelle, J. Bekaert, and V. V. Moshchalkov, Phys. Rev. B 70, 094503 (2004).

${ }^{22}$ I. V. Grigorieva, L. Y. Vinnikov, W. Escoffier, J. Richardson, S. V. Dubonos, V. Oboznov, and A. K. Geim, cond-mat/0506642.

${ }^{23}$ To separate multivortex states from giant vortex states, we used the following criterion: When the maximum Cooper-pair density in between two vortices becomes larger than $10^{-2}$, we define the state as a multivortex state.

${ }^{24}$ In Fig. 4, for all samples, there are more states in decreasing magnetic field than in increasing field. This is because for some reasons the voltage in increasing field is noisier than that in decreasing fields, making the identification of the transition points more difficult in increasing field.

${ }^{25} \mathrm{M}$. Tinkham, Introduction to Superconductivity, 2nd ed. (McGraw-Hill, New York, 1996).

${ }^{26}$ D. Bertrand, Ph.D. thesis, Université Catholique de Louvain, Belgium, 2005.

${ }^{27}$ B. J. Baelus, K. Kadowaki, and F. M. Peeters, Phys. Rev. B 71, 024514 (2004). 